\title{
Mosaic growth of diamond: a study of homoepitaxial flame deposition and etching of $\{001\}$-oriented diamond layers
}

\author{
J.J. Schermer * , F.K. de Theije, L.J. Giling \\ Research Institute of Materials, Unicersity of Nijmegen, Toernooileld, 6525 ED Nijmegen. The Netherlands
}

Received 20 November 1995: accepted 6 December 1995

\begin{abstract}
This study describes homoepitaxial growth and etch phenomena observed on cubic diamond faces as obtained by the application of an acetylene-oxygen combustion flame. It is demonstrated that flame etching of the diamond substrates prior to growth decreases the number of crystallographic imperfections in the diamond layers especially at the edges. This enables a more uniform distributed step generation by the large number of individual dislocations present in the used natural type IIa diamond substrates. Due to this improvement it appears possible to grow one single crystal diamond layer on top of several closely spaced, well-aligned natural diamond substrates. In the present work the overgrowth of a three-piece mosaic structure with gaps along $\langle 100\rangle$ as well as along $\langle 110\rangle$ is reported for the first time. The single crystal nature of this flame-deposited diamond layer could be confirmed by the observation of continuous growth step patterns at the surface across the joint regions. The widths of the gaps between the substrates in the original mosaic structure varied from 7 to $25 \mu \mathrm{m}$, which is more than an order of magnitude larger than the gaps along $\langle 100\rangle$ in two-piece mosaic structures, of which the single crystal overgrowth by HFCVD was previously reported. Comparison of micro-Raman spectra obtained from the joint regions with those from other parts of the overgrown mosaics discussed in the present work, show a larger FWHM of the diamond peak in case of a $\langle 110\rangle$ joint and a shift of the peak position to higher wave numbers in case of a $\langle 100\rangle$ joint.
\end{abstract}

\section{Introduction}

By now it is well known that CVD diamond films grown via the $\{001\}$ faces are most suited for future applications in optics and semiconductor technology. This is because they reveal the lowest concentrations of point and extended defects [1-4]. Therefore, in recent years the deposition of $\{001\}$ textured layers either directly on silicon and silicon-carbide substrates [5-8], or on a large number of small aligned diamond seed crystals $[9,10]$, has gained more and

Corresponding author. Fax: +31243652314. more interest. An alternative method to produce large area $\{001\}$ diamond plates, is by homoepitaxial deposition of a continuous layer on top of a mosaic structure consisting of several medium-sized, closely spaced and well-aligned diamond substrates [11,12]. Once such a layer can be made sufficiently thick, several large area $\{001\}$ diamond plates might be obtained from it by the application of lift-off [13-15] and splicing techniques.

The aim of this study is to demonstrate the possibility to overgrow a mosaic structure of several millimetre-sized seed crystals by one relatively thick single crystal diamond layer. For this purpose the exact and near exact cubic faces of a fairly large 
number of single crystals, which were deposited by the acetylene-oxygen combustion flame technique $[16,17]$, will be studied. The specimens were homoepitaxially grown on natural type IIa diamond substrates of which some already had a relatively thin epitaxial layer obtained by hot-filament-assisted chemical vapour deposition (HFCVD) [3,11]. Previous infra-red (IR) and Raman analyses indicated that these diamond layers can be classified as type IIa [2]. Flame growth took place at deposition temperatures $\left(T_{\mathrm{d}}\right)$ ranging from 1050 to $1200^{\circ} \mathrm{C}$ and acetylene supersaturations $\left(S_{\text {ac }}\right)$ between $4 \%$ and $5 \%$. Within this range no clear relation was found between the growth conditions and the growth rate or the surface morphology. Rather than the growth conditions, the presence of crystallographic imperfections seem to determine the features of the grown layers as will be addressed in detail in the present study. Besides growth in the acetylene feather, the oxidizing outer part of the combustion flame offers the possibility to etch diamond $[18,19]$. It will be demonstrated that flame etching of diamond substrates prior to growth decreases the number of crystallographic imperfections in the homoepitaxial diamond layers especially at the edges so that the overgrowth of mosaic structures by one single crystal diamond layer becomes possible.

The surface topography of the samples was analyzed by differential interference contrast microscopy (DICM) and scanning electron microscopy (SEM). Information about the incorporation of impurities was obtained by cathodoluminescence (CL) and micro-Raman spectroscopy.

\section{Macrostep patterns on $\{001\}$ diamond faces}

Characteristic for flame-deposited diamond layers on exact and near exact $\{001\}$ substrates is the presence of macrostep patterns as shown in Fig. 1 [2,20-22]. Such step bunches originate from a pile-up of atomically high steps generated at a given step source [23]. It is not likely that the pattern of macrosteps sometimes with wide terraces in between is caused by variations in the step generation rate at the source. Therefore, it is assumed that this pile-up occurs because the propagation of individual, low steps is hampered by impurities from the gas phase

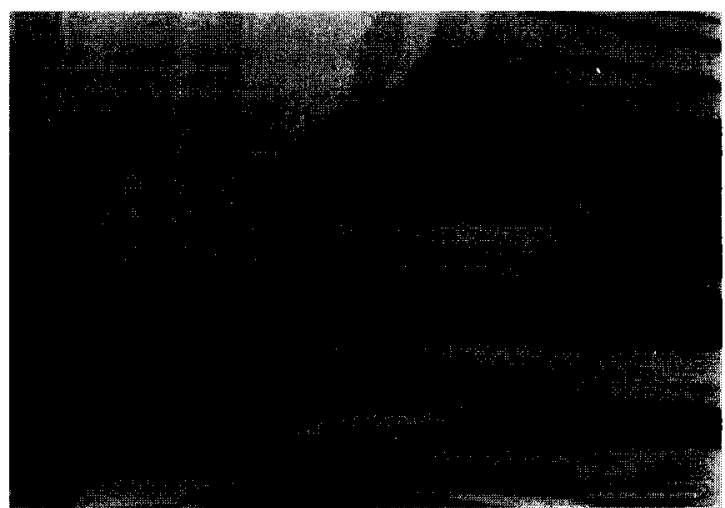

Fig. 1. SEM image of macrosteps on a (001) top face of a flame-grown single crystal $\left(T_{\mathrm{d}} \approx 1150^{\circ} \mathrm{C}, S_{\mathrm{ac}} \approx 4 \%\right)$ taken at an angle of $45^{\circ}$. The steps along the $\langle 110\rangle$ directions, as indicated by the arrows, are smooth and more or less straight while those in other directions have a rough, pitted appearance.

adsorbed at the terraces in front of them [24,25]. Recently it was demonstrated that macrostep bunches also occur on $\{001\}$-oriented HFCVD-grown layers upon the addition of nitrogen in the gas phase [7]. From this it can be concluded that the impurities blocking the propagation of the individual steps along the diamond surface are most probably nitrogen containing species which, in the case of flame deposition, diffuse into the acetylene feather from the ambient $[22,26]$. On the other hand it was found that the overall growth rate of the $\{001\}$ faces increases upon nitrogen addition $[7,27]$. Possibly nitrogen containing species adsorbed at the surface also act as sites for two-dimensional nucleation of new layers at the terraces in between the macrostep bunches (birth and spread).

In accordance with the PBC theory [28] and the $(2 \times 1)$ reconstruction of the surface [29], the macrosteps on exact $\{001\}$ diamond layers have a tendency to become aligned parallel to the $\langle 110\rangle$ directions (see Fig. 1) $[2,18,20]$. DICM observations reveal that these macrosteps have a smooth surface with more or less discrete inclinations ranging from $5^{\circ}$ to $20^{\circ}$ with respect to the substrate $[2,30]$. From this it can be deduced that the surfaces of these steps correspond to orientations with Miller indices $\{h h k\}_{h<k}$ between $\{001\}$ and $\{113\}$. Recently, the existence of a discrete number of orientations in this zone with a minimum in surface energy was explained by a difference in the possibilities for these 
orientations to lower their surface free energy by dimer formation [30]. Substrates with an orientation slightly off from the exact $\{001\}$ already exhibit a large number of individual steps before growth which develop to macrosteps during deposition. In case that these macrosteps are not aligned along $\langle 110\rangle$, they were observed to have a rough appearance as can also be observed in small regions of the layer shown in Fig. 1. It seems that the steps break up into small perpendicular steps aligned along $\langle 110\rangle$, corresponding to orientations between $\{001\}$ and $\{113\}$ with a lower surface free energy.

Examination by CL topography reveals that areas at the $\{001\}$ surfaces with well-developed steps along $\langle 110\rangle$ mainly reveal blue band A emission [31] indicative for highly pure diamond. The orange-red $575 \mathrm{~nm}$ system, characteristic for nitrogen incorporation as nitrogen-vacancy pairs [32,33], dominates the luminescence of areas with steps which are aligned along other directions. It has been suggested that band A luminescence originates from electronhole recombination at donor-acceptor pairs [31]. In this the donor might be an aggregate of an even number of substitutional nitrogen atoms [34] with boron as acceptor $[35,36]$. On the other hand it was argued that band A in CVD diamond is caused by dislocations [32,37,38], in which case the CL is expected to be polarized with the $E$-vector parallel to the dislocation line $[39,40]$. The presence of nitrogen in the flame-grown single crystals examined in the present work can be explained by in-diffusion from the ambient. The incorporation of boron, however, is highly unlikely since there never was a boron source in, or near the flame set-up used. Therefore, the blue emission of the flame-deposited single crystals, which can be observed in case of a relatively low nitrogen impurity level, is most probably dislocation related. The observed inhomogeneity in the $\mathrm{CL}$ of the cubic faces is most readily explained by step-geometry-related differences in impurity incorporation. Steps along $\langle 110\rangle$ have the most stable configuration so that the uptake of impurities is generally quite low and blue band A luminescence can be observed. Steps along other, energetically less favourable, directions are at least partly kinked thus impurities are relatively easily trapped in the growing diamond film, giving rise to the orange-red $\mathrm{N}-\mathrm{V}$ related emission. This correlation between the incorporation of impurities, in particular nitrogen, and the misorientation of the substrate is beautifully illustrated by a previously described homoepitaxial sphere growth experiment $[21,30]$.

\section{Sources of macrosteps on $\{001\}$ diamond faces}

If a crystal face grows via a layer mechanism like the diamond $\{001\}$ face, two boundary conditions need to be fulfilled. In the first place there needs to be a supersaturation of growth species near the surface of the crystal. In the second place, steps or a source of steps is needed. The most active step source determines the growth rate of the layer. During flame deposition, a supersaturation of growth species is obtained by the use of a fuel-rich gas mixture. The surplus of acetylene, typically between $3 \%$ and $6 \%$, is decomposed in all kinds of active hydrocarbon species by the energy released in the primary combustion process [41]. These species reach the diamond surface by active transport in the main stream, which has a stagnation flow geometry, and diffusion through the boundary layer above the substrate [42-44]. A thin boundary layer decreases the diffusion time thus results in a high flux of growth species towards the growing surface. In case of homoepitaxy on relatively small (1-4 $\mathrm{mm}$ in diameter) diamond seed crystals, the thickness of the boundary layer can be assumed to be fairly constant over the entire surface with exception of the substrate rims. At the rims of the substrates, which are between 0.2 and $0.5 \mathrm{~mm}$ in thickness, the main flow starts to bend down towards the substrate holder resulting in a decrease in the boundary layer thickness. Consequently, the edges encounter a higher concentration of growth species than the rest of the substrate.

Step sources on the cubic diamond surface were found to be substrate misorientations from the exact $\{001\}$ orientation, screw dislocations outcropping the substrate surface, foreign (diamond) particles, penetration twins and imperfections at the edges of the substrate [23]. During deposition, several of these sources give rise to the formation of a growth hillock because they continuously create steps at a higher rate than their surroundings.

Fig. 2a shows a growth hillock obtained by join- 

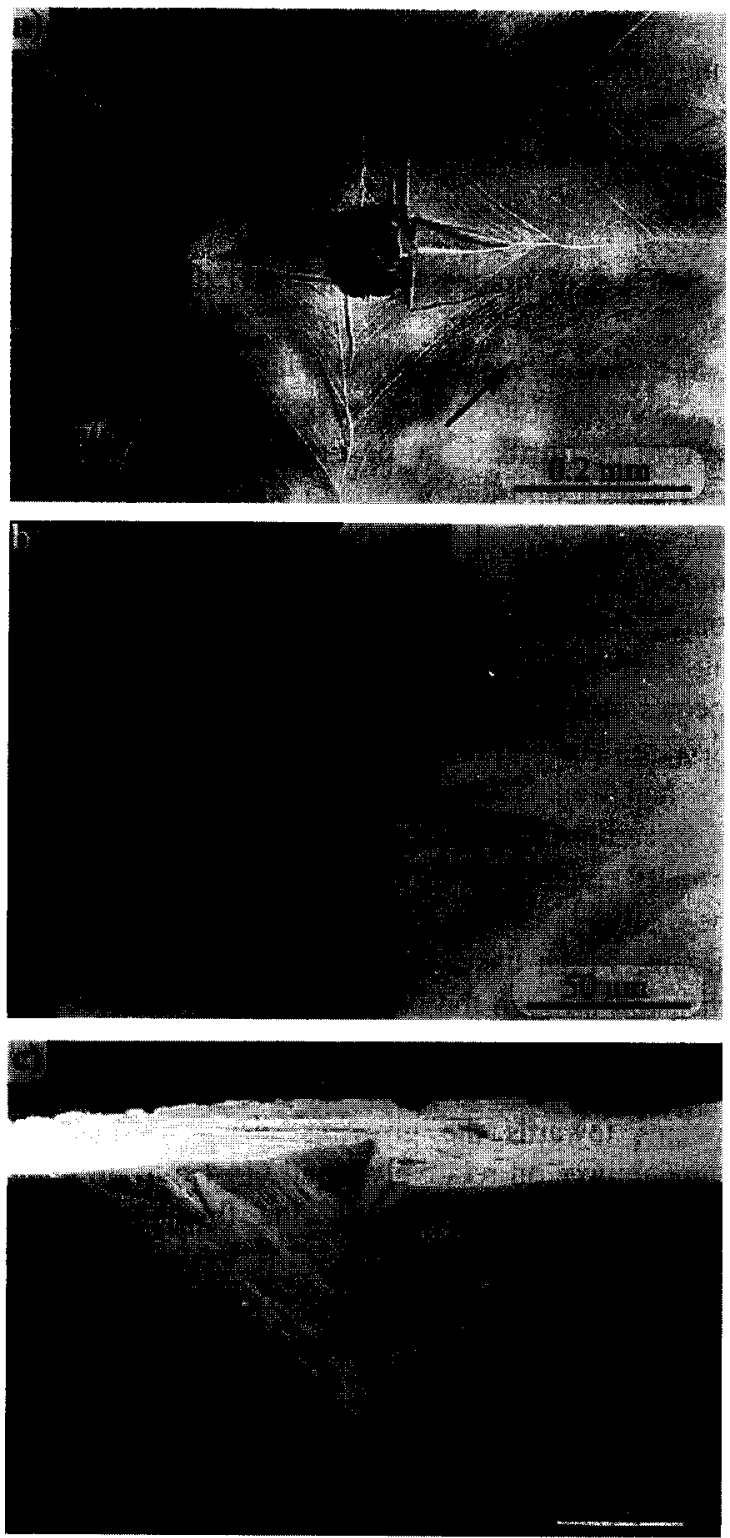

Fig. 2. Step-emitting imperfections at the cubic top faces of flame-grown single crystals $\left(T_{\mathrm{d}} \approx 1150^{\circ} \mathrm{C}, S_{\mathrm{ac}} \approx 4 \%\right)$. DICM images of (a) a foreign (diamond) particle with several discrete points of step generation and (b) a bundle of dislocations located straight above the edge of the original substrate (arrows indicate the [110] directions). (c) SEM image of a cusp on the edge of two adjacent $\{100\}$ faces.

ing of steps generated by contact nucleation at several discrete points of a foreign particle. The presence of re-entrant corners between the particle and the $\{001\}$ surface lowers the activation barrier for two-dimensional nucleation. Generally the top of such a hillock is ill-defined due to the close grouping of randomly oriented diamond particles and penetration twins [6,45]. Hillocks like these appear in large numbers on HFCVD-grown $\{001\}$ samples $[4,21,46,47]$ but at most a few develop on flamegrown cubic faces [20,22].

Another group of hillocks, which most abundantly are located near the edges of the $\{001\}$ samples is characterized by sharp, point-shaped summits free from foreign particles and twins (see Fig. 2b). Close examination shows that in most cases the summits of these elevations are located straight above the original edges of the substrate. This indicates that the step sources are linear defects, which propagate perpendicular to the $\{001\}$ surface during growth. Due to minimization of their line energy, dislocations in many crystals tend to end perpendicular to a growth face [48]. Therefore, the point-tipped hillocks most probably are not due to foreign particles but originate from a large number of closely spaced, cooperating dislocations with a net screw component of the Burgers vector perpendicular to the surface. Close to the top of the hillock, just after emission, the steps are low and equally spaced, further away they pile-up and form macrosteps. A large number of these hillocks located near the rim of the $\{001\}$ crystal face with an adjacent $\{111\}$ face reveal a line-shaped feature from their summit to the edge of the cubic face (see Fig. 2b). This is one small end of a crack which with its full length is located in the growth sector of the $\{111\}$ side face.

On the edges of most cubic faces at the intersection lines with adjacent $\{100\}$ side faces, "cusps" can be observed (see Fig. 2c). These are re-entrant corners bounded by two $\{111\}$ faces and usually are accompanied by penetration twins. They probably originate from locations where, due to cutting and polishing, sub-micron parts of the substrates were chipped out along the $\{111\}$ cracking planes of diamond. Locally this process is accompanied by nanofractures and strain, which probably causes the development of twins in the cusps. During growth the cusps, which act as step sources, form elevations with the shape of half a growth hillock on both the adjacent $\{001\}$ facets (see Fig. 3).

For flame-grown layers on exact $\{001\}$ substrates the distribution and activity of the step sources, 

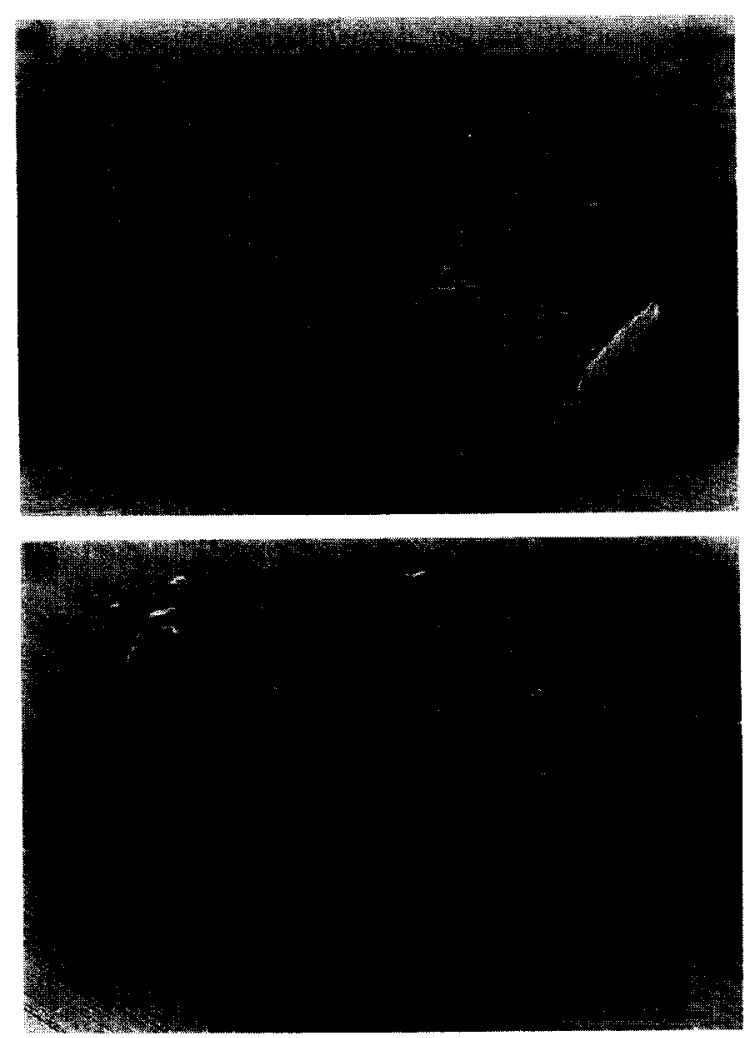

Fig. 3. SEM overviews of single crystals deposited on (a) a nearly exact (001) diamond substrate and (b) on a (001) substrate misoriented $4^{\circ}$ towards [ 100$]\left(T_{\mathrm{d}} \approx 1200^{\circ} \mathrm{C}, S_{\mathrm{ac}} \approx 4 \%\right)$. The images were taken with the same magnification at an angle of $55^{\circ}$ with the normal at the top face.

which in fact are crystallographic imperfections, is not uniform over the surface. From the previous description of these growth centres it is clear that most of them are located near the periphery of the cubic face. Furthermore, the sources close to the edges of the sample are more active than the central sources since growth precursors can reach them by diffusion through the locally relatively thin boundary layer from above as well as from aside. Sometimes more centrally located hillocks are generated by foreign particles, but most times such particles, if not blown away by the large gas flow when the sample was brought into the flame, are rapidly overgrown by steps from sources at the edges. Frequently these particles can be observed as inclusions at the interface by focusing into the transparent diamond layer with an optical microscope, which demonstrates the capability to overgrow defects by the step bunches generated during flame deposition. Due to the nonuniform distribution and activity of the described step sources, the final shape of $\{001\}$ layers grown on more exactly oriented substrates reveal a number of crystallographic imperfections at their edges and often obtain a concave shape (see Fig. 3a). For flame-grown samples on misoriented substrates, steps resulting from the misorientation compete with the step sources near the edges and less concave or even straight layers develop as shown in Fig. 3b.

\section{Pre-deposition etching of $\{001\}$ diamond sub- strates}

Besides growth, the acetylene-oxygen torch also offers the possibility to etch diamond in the oxidizing outer flame. This zone is located outside the acetylene feather in case of a fuel-rich flame or directly outside the inner cone of an oxygen-rich flame. Natural type IIa diamond substrates etched for a few minutes at circa $950^{\circ} \mathrm{C}$ reveal a large number of square etch pits (see Fig. 4) with their rims along $\langle 110\rangle$, which is consistent with the $2 \times 1$ reconstruction of the surface $[18,29,30]$. These pits with density between $10^{6}$ and $10^{8} \mathrm{~cm}^{-2}$, probably correspond to dislocations in the substrate [49], which for this type of diamond typically have a density in the same order of magnitude [50]. All these dislocations are potential step sources which, in the absence of the

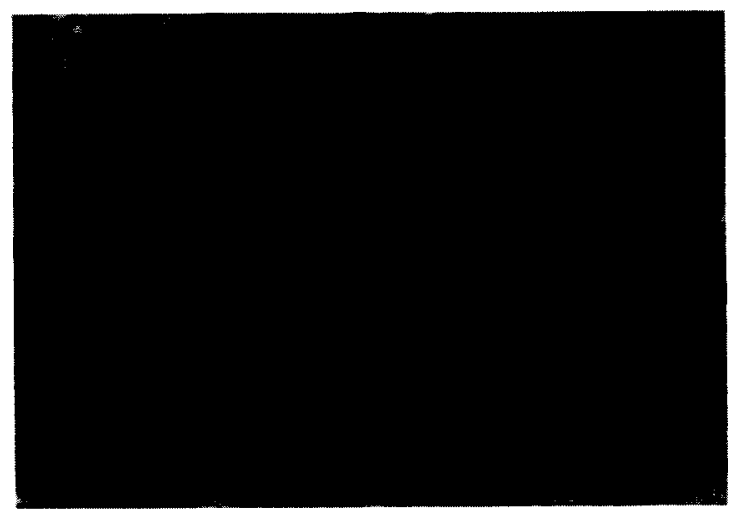

Fig. 4. SEM image of etch pits on a (001) type IIa natural diamond substrate etched for two minutes at $975^{\circ} \mathrm{C}$ and a supersaturation of $-2 \%$ in the oxidizing outer flame. The arrow indicates the $[110]$ direction. 
small number of discrete sources with a high activity, might give a more or less uniform deposition rate over the entire surface. After prolonged etching the pits overlap resulting in a rough appearance of the surface.

Experiments demonstrated that by the application of an etching procedure prior to growth, the occurrence of large elevations like cusps and hillocks on flame-grown samples can be prevented or at least strongly reduced. This can be explained by the fact that a number of highly active step sources like foreign particles are etched away relatively easy. Furthermore, the etching process is more effective close to the rims of the substrates which become more rounded. By this, most of the sub-micron chip- out is removed and local misorientations towards the rims of the cubic face are obtained. Due to these local misorientations, steps from the single dislocation sources in the centre have the opportunity to overrun the remaining sources at the rims. As a result the macrosteps propagating from the centre towards the edges give the $\{001\}$ faces, which are obtained in relatively short deposition experiments (less than one hour), a flat or even slightly convex shape. This direction of step propagation has the additional advantage that contamination accumulating in front of the steps, is driven towards the rims of the specimens rather than being trapped in the centre of the $\{001\}$ face, where it might cause cracking of the epitaxial layer [51]. After longer deposition times,
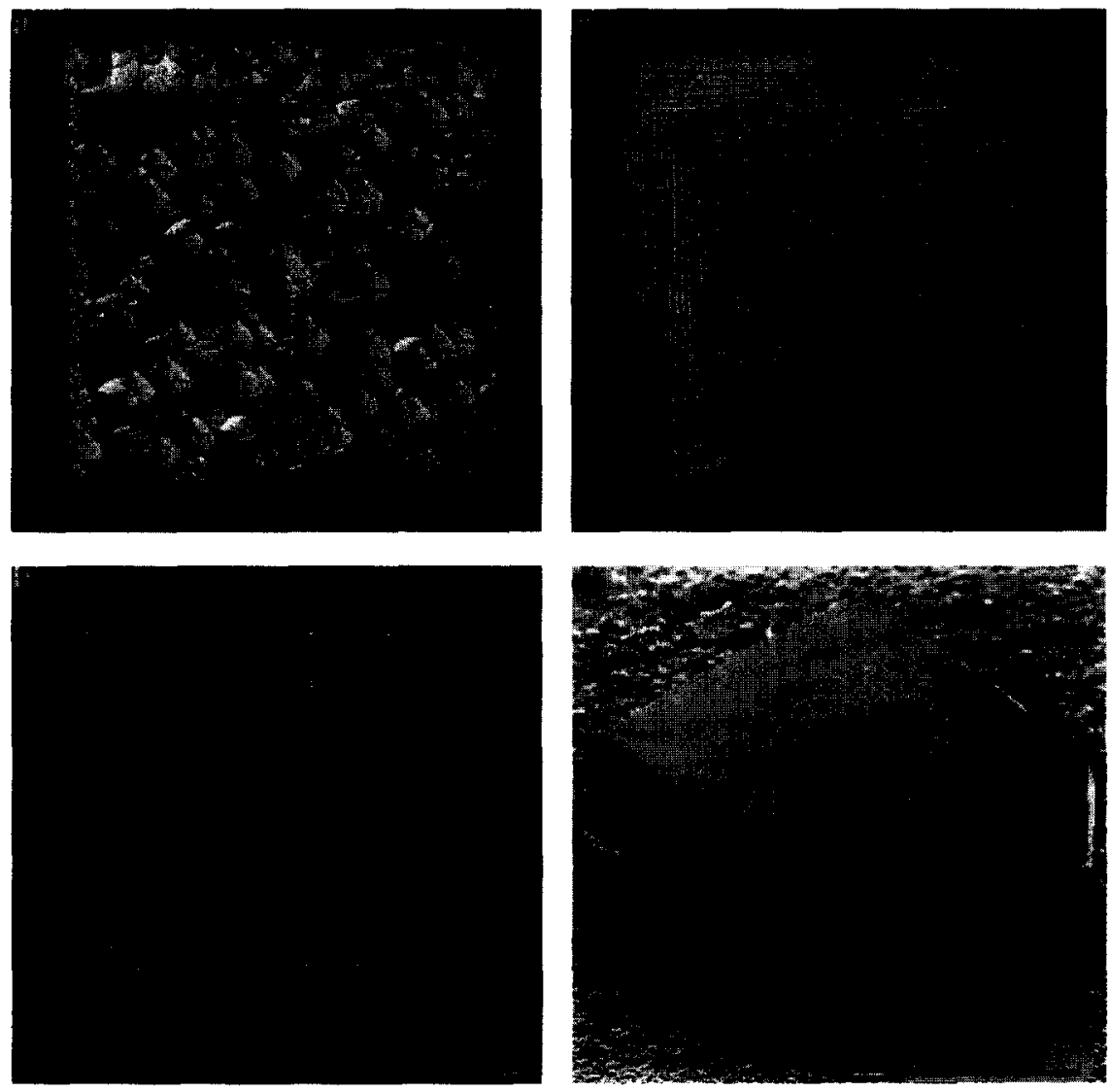

Fig. 5. The surface morphology (DICM) and an overview (SEM) of a diamond single crystal in different stadia of homoepitaxial growth on a (001)-oriented substrate. (a) The initial diamond film obtained by HFCVD in which the arrow indicates the [110] direction. (b) The sample after etching in the oxidizing outer flame and sequential growth of ten minutes in the feather of an acetylene-oxygen flame. (c), (d) The final specimen after flame deposition of $100 \mathrm{~min}$ in total. The overview, taken at an angle of $50^{\circ}$ with the normal at top face, shows the "chipped out" part in one of the $\{11 \mathrm{I}\}$ side faces. (a), (b) and (c) are on the same scale. 
the edges usually become straight and start to act as a step source along the full length, but large imperfections at the rims (see Fig. 3) are rarely encountered on such specimens (see Figs. 5 and 6 and 8). However, in some cases, if deposition takes place relatively close (less than $1 \mathrm{~mm}$ ) to the flame front, the propagation direction of the macrosteps from the centre towards the edges can be maintained even after growth experiments exceeding six hours. This indicates that the concentration of growth species in the centre of the sample has been higher than at the edges, which indeed is to be expected with the sharp-tipped laminar flame front at close distance $[22,41,44]$. Unfortunately, specimens obtained under such violent conditions reveal extreme variations in layer thickness, e.g. from 250 to $500 \mu \mathrm{m}$ and usually develop a large number of step-emitting imperfections during growth.

The advantage of the pre-deposition etch procedure in a flame-deposition experiment can be clearly demonstrated using a natural diamond substrate with a HFCVD epitaxial diamond film on top of it. Exact or near exact cubic diamond films obtained by HFCVD exhibit a high density $\left(10^{4}-10^{5} \mathrm{~cm}^{-2}\right)$ of hillocks (see Fig. 5a), mostly nucleated at foreign particles but also at bundles of dislocations outcropping the substrate surface $[4,46,47]$. The asymmetrical shape of the hillocks on the (001) top face of the specimen shown in Fig. 5a indicates that the original substrate had a slight misorientation of maximal $2^{\circ}$ towards [110]. This misorientation was not large enough to prevent the development of growth hillocks
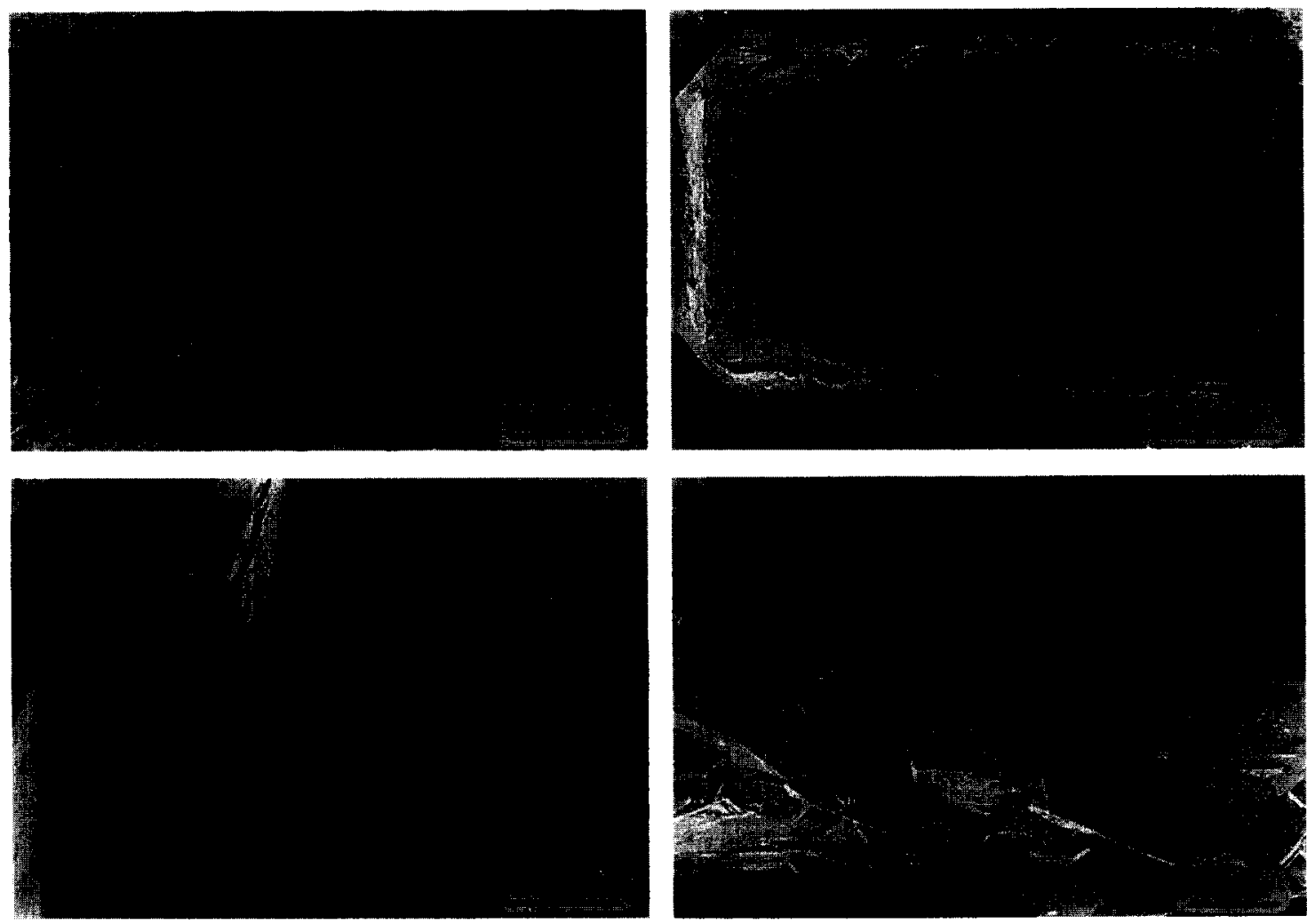

Fig. 6. SEM images of flame-grown diamond layers on top of two different sets of two (001)-oriented natural diamond seed crystals with a gap in the [110] direction. (a) Overview of a mosaic growth experiment on seed crystals with a relatively large crystallographic misalignment with respect to each other and (b) detail of the step-like feature at the joint region showing a discontinuity at the bottom, both taken at an angle of $55^{\circ}$ with the normal at the top face. (c) Overview and (d) detail of another mosaic growth experiment showing slightly deteriorated $\{111\}$ facets in the gap which obstructed the formation of one single crystal top face. 
and three-dimensional nuclei on the initial HFCVD film, for which a misorientation of at least $4^{\circ}$ is needed [4]. The film, which originally was about 10 $\mu \mathrm{m}$ in thickness, exhibited cracks nearby and parallel to the $\langle 110\rangle$ edges of the (001) top face. A small piece of the film and underlying substrate was even chipped out along such a crack. The fact that the fracture patterns did not interfere with the surface morphology of the film indicated that most cracking occurred after cessation of the growth by HFCVD.

After an etching period of twelve minutes in the acetylene-oxygen flame $\left(T_{\text {etch }}=960^{\circ} \mathrm{C}, \quad S_{\mathrm{ac}}=\right.$ $-2 \%$ ), the layer revealed a small number of remainders from the larger hillocks on a roughened surface caused by overlapping etch pits. Successive growth of the sample for only ten minutes by the flame technique $\left(T_{\mathrm{d}}=1050^{\circ} \mathrm{C}, \quad S_{\mathrm{ac}}=5 \%\right)$ completely changed the structure of the diamond layer (see Fig. $5 b)$. From its appearance it can be deduced that the slight misorientation of the sample has been the main step source. The remainders of the hillocks from the original HFCVD film were fully overrun by these steps and do not act longer as a source themselves. Even the cracks present close to the edges of the HFCVD layer were overgrown.

Another $1.5 \mathrm{~h}$ of flame deposition at the same sample using the same conditions resulted in a homoepitaxial layer with a total thickness of about 90 $\mu \mathrm{m}$. Fig. 5c shows that three of the four substrate edges have acted as a step source, emitting large steps along the full dimensions of the (001) face, but do not reveal imperfections. The dimensions of the cubic top face have increased with respect to that in the earlier stages of the experiment and does not show the area of which a piece was chipped out. The SEM micrograph in Fig. 5d reveals that the area with the chip-out is now located in a $\{111\}$ side face of the sample. From the relative dimensions of the $\{111\}$ and $\{100\}$ side faces of the diamond crystal (see Fig. 5d) the ratio of the growth rates in the $\langle 111\rangle$ and $\langle 001\rangle$ directions was calculated to be about 1 . This value is low enough to get lateral expansion of the $\{001\}$ faces during growth [45,52]. Due to this expansion and the well-shaped edges it becomes possible to overgrow several closely spaced $\{001\}$-oriented diamond seed crystals by a continuous diamond layer as will be demonstrated in the next section.

\section{Enlargement of $\{001\}$-oriented diamond layers by mosaic growth}

The dimensions of natural diamond seed crystals are rather limited, which restricts the dimensions of $\{001\}$-oriented layers obtained by homoepitaxy on individual seed crystals. This explains why the deposition of $\{001\}$ textured layers on non-diamond substrates has gained more and more interest in the past few years. Although $\{001\}$ textured diamond films are promising material for a number of applications, at this moment even epitaxially textured films have a relatively large angular spread of the diamond crystal orientations and contain a high density of (low-angle) grain boundaries [6,53]. Therefore, these films are less suited for high quality optical components or as substrates for active electronic devices. The most direct way to enlarge the dimensions of $\{001\}$-oriented single crystal substrates is by the deposition of a continuous diamond layer on top of a mosaic structure of two or more medium-sized closely spaced natural diamond seed crystals $[11,12]$.

\subsection{Non-single crystal overgrowth of mosaics}

For a preliminary investigation of mosaic growth by the flame technique, a number of $1 \times 1 \times 0.5$ $\mathrm{mm}^{3}$ seed crystals with $\{001\}$ top and bottom faces and $\{110\}$ side faces were obtained from a commercial supplier [54]. Two by two these crystals were soldered in close proximity to each other onto a molybdenum substrate by a high temperature soldering material (melting point $\sim 1250^{\circ} \mathrm{C}$ ) and overgrown by flame deposition after an initial etch period. Fig. 6a shows a typical result of such an experiment in which a gap of about $50 \mu \mathrm{m}$ was bridged in about $6 \mathrm{~h}$ and $45 \mathrm{~min}$ by deposition of a $200 \mu \mathrm{m}$ thick diamond layer $\left(S_{\mathrm{ac}} \approx 4 \%, T_{\mathrm{d}} \approx\right.$ $1100^{\circ} \mathrm{C}$ ). Close to the joint region the deposited layer exhibits a large step of about $30 \mu \mathrm{m}$ in height, which has a rough appearance. The position and the roughness of this feature, which appeared in most of these experiments, makes it difficult to obtain evidence, which indicates that the entire grown layer is one single crystal instead of two crystals with a grain boundary. In fact, in this particular case it was relatively easy to demonstrate that the deposited layer is not one single crystal because SEM analysis 
under the appropriate angle revealed a clear discontinuity at the bottom of the step-like feature (see Fig. $6 b)$. So rather than a step bunch on a single crystal surface, this feature is the side face of one of the crystals, which has partly grown over the top face of the other. The presence of the grain boundary is related with the large in-plane misalignment of the substrate orientations of about $10^{\circ}$ with respect to each other as could be deduced from the relative orientations of the exact $\{100\}$ side faces which have developed at the corners of both substrates during deposition. The misalignment in crystallographic orientation despite the fact that the sides of the substrates at both sides of the gap were parallel to each other occurred because, according to the vendors information, the orientations of the polished substrate faces normally are much less than within $\pm 1^{\circ}$. This makes it virtually impossible to overgrow a parallel aligned set of such substrates by one single crystal.

Despite the presence of a grain boundary, spectroscopic measurements obtained from the region at the joint do not reveal large differences with those obtained from other regions of the top layer. Roomtemperature cathodoluminescence topography of the mosaic showed hardly any emission from the main parts of the top surface, while the most pronounced step bunches and $\{111\}$ side faces revealed blue band A emission. Raman spectra obtained from different spots at the top face revealed no other features than a sharp diamond peak at $1332 \mathrm{~cm}^{-1}$ with a full width at half maximum (FWHM) of about $2.8 \mathrm{~cm}^{-1}$. As expected the FWHM of the peak in the spectra obtained at the joint was 0.1 to $0.3 \mathrm{~cm}^{-1}$ larger due to the presence of an extended number of defect sources [12] but no shift of the peak position was observed. The diamond peak in a spectrum taken at the step, or rather the $\{111\}$ side face of the one single crystal, which has partly overgrown the other (see Fig. 6b), also has the same position but a FWHM of $3.4 \mathrm{~cm}^{-1}$. Despite the fact that the deposited layer is not one single crystal, both seed crystals appeared well connected after removal of the molybdenum substrate by etching in a boiling mixture of $\mathrm{HCl}$ and $\mathrm{HNO}_{3}$.

The described features for the sample shown in Figs. $6 a$ and $6 \mathrm{~b}$ were also encountered on the other mosaics, although one of them, for which the gap was as large as $70 \mu \mathrm{m}$, does not exhibit a step-like feature so that the joint region is easy accessible for visual inspection. Fig. $6 \mathrm{c}$ shows that two pronounced \{111\} facets had developed in the gap at the rims of the seed crystals during the $4 \mathrm{~h}$ and $45 \mathrm{~min}$ of deposition $\left(T_{\mathrm{d}} \approx 1150^{\circ} \mathrm{C}, S_{\mathrm{ac}} \approx 4 \%\right)$. After prolonged exposure to the gas phase, $\{111\}$ facets always suffer from twin formation and secondary nucleation $[22,26]$. Once this process has started, the deposit at the joint will become more and more polycrystalline in nature and obstruct the formation of one single crystal on top of the mosaic. Fig. 6d shows that in the present case the $\{111\}$ facets in the gap are already slightly deteriorated at the grain boundary separating both crystals. So the deposited layer is not expected to be one single crystal although the within circa $2^{\circ}$ in-plane substrate alignment is much better than for the sample shown in Figs. $6 \mathrm{a}$ and $6 \mathrm{~b}$. From this experiment it might be concluded that the maximum [110] gap that can be allowed is limited to a width which at least is below $70 \mu \mathrm{m}$. Related with the deterioration of the $\{111\}$ facets at the joint, the FWHM of the diamond peaks in Raman spectra from this region were 0.2 to $0.6 \mathrm{~cm}^{-1}$ larger than the 2.6 $\mathrm{cm}^{-1}$ of the peaks in the spectra obtained from the centre of each specimen.

A similar experimental approach as described in this section was used by Malta and coworkers [12] to enlarge the $\{001\}$ diamond surface using type Ia diamond substrates with $\{100\}$ side faces obtained from the same manufacturer [54] as those used in the present study. After growth by RF-plasma-enhanced CVD, they observed features similar as described above, like a slight increase in the FWHM of the diamond Raman peak and a step-like feature at the joint, and claim epitaxial joining of the seed crystals. Unfortunately, they did not report any evidence for this statement but fractured the obtained mosaic structure at the joint in the cause of their further investigations.

\subsection{Single crystal overgrowth of mosaics}

It is clear that if no particular attention is paid to the crystallographic alignment of the seed crystals the deposited top layer in general is not one single crystal. To control the alignment, the orientation of the individual seed crystals must be measured by an $\mathrm{X}$-ray diffraction or an electron beam diffraction 
technique and one must be able to correct the positioning of each substrate without disturbing the rest of the mosaic. This requires a huge technical effort. However, to demonstrate the feasibility of the mosaic growth process, a set of highly oriented diamond substrates might be obtained by cutting a single polished diamond plate in several pieces and re-assembling them close to each other at their original position. Recently, two different mosaics obtained in this way were successfully overgrown by one single crystal top layer in our laboratory using the hot-filament technique [11]. For these experiments two $4 \times$ $2 \times 0.5 \mathrm{~mm}^{3}$ diamond plates with $\{100\}$ side faces were cut along [100] in two $2 \times 2 \times 0.5 \mathrm{~mm}^{3}$ pieces each. The (001) top faces of both plates were misoriented by about $6^{\circ}$ towards [010] and [100], i.e. perpendicular respectively parallel to the direction of the cut. The two pieces of each set were soldered onto a molybdenum plate at a distance less than 1 $\mu \mathrm{m}$ next to each other and overgrown by HFCVD films of 14 and $40 \mu \mathrm{m}$ in thickness. Cracks in the deposited top layers running across the original interface between the two seed crystals provided evidence that in both cases one single crystal was grown on top of two substrates. A second characteristic feature of the mosaics was the presence of a band of enhanced growth parallel to the original gap between both elements. These bands, which originated from a large number of step sources at the overgrown gap, were shifted by 100 and $500 \mu \mathrm{m}$ relative to the gap in the direction of the misorientation of the substrates.

For the present study the acetylene-oxygen flame was applied to deposit additional single crystal layers exceeding $250 \mu \mathrm{m}$ in thickness on top of these pre-grown mosaics. The deposition of these layers was preceded by only one minute of etching in the outer flame beyond the acetylene feather, to prevent the total etching of the single crystal films bridging the initial gaps. After flame deposition no cracks or bands of extended growth were found at the surface of the final layer, which once more illustrates the ability of this technique to overgrow defects like cracks on $\{001\}$-oriented substrates. Fig. 7a shows one of the two mosaics for which a large part of the top face located at the left-hand side has become more or less exact in orientation during the $7 \mathrm{~h}$ of flame growth $\left(T_{\mathrm{d}} \approx 1050^{\circ} \mathrm{C}, S_{\mathrm{ac}} \approx 5 \%\right)$. The exact
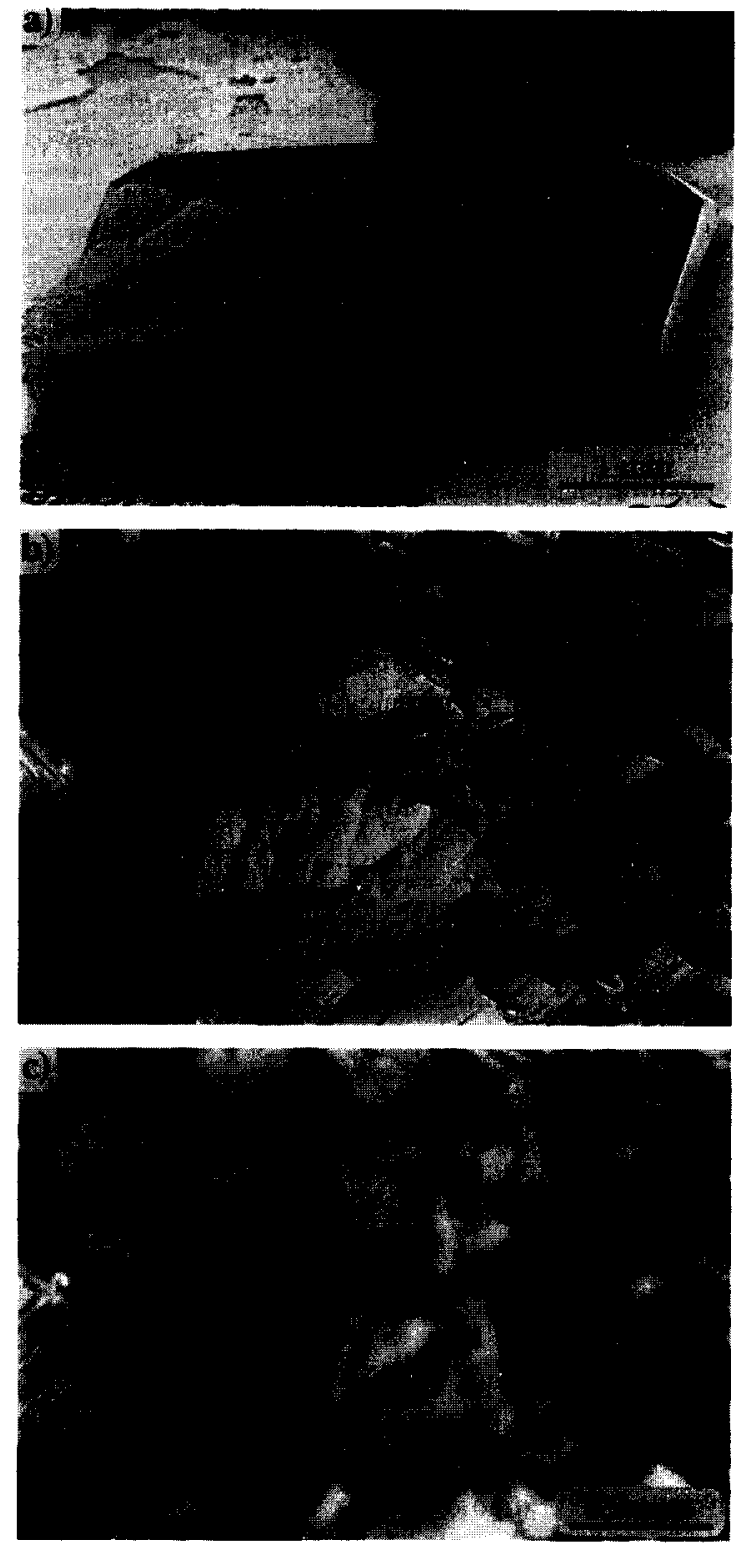

Fig. 7. Image of one flame-deposited single crystal diamond layer on a by the hot filament technique pre-grown mosaic of two perfectly aligned seed crystals with (001) top faces. (a) SEM overview taken at an angle of $55^{\circ}$ with the normal to the top face. (b) Surface and (c) sub-surface DICM images of the same area at the joint region in which the arrow indicates the original gap in the $[100]$ direction.

area, which has a relatively smooth appearance, is dominated by several large hillocks generated at foreign particles. These hillocks were already present 
at the $14 \mu \mathrm{m}$ thick layer obtained by HFCVD and obviously were not sufficiently removed by the short etching procedure. The right-hand side of the specimen is still dominated by roughly shaped step bunches along [100] resulting from the $6^{\circ}$ misorientation of the mosaic substrates in the [010] direction. The exact and misoriented parts of the deposited layer are separated by a V-shaped step bunch with a
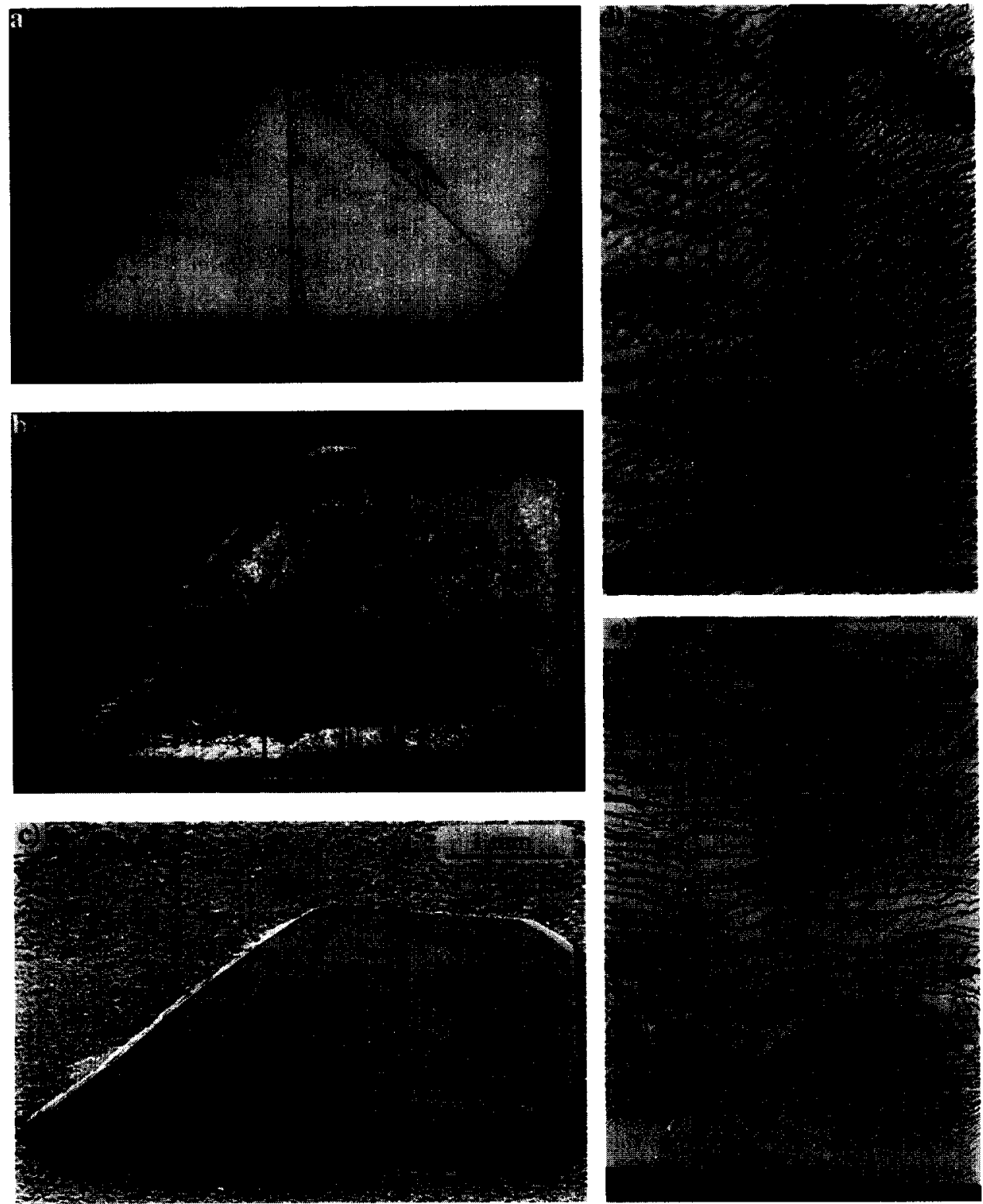

Fig. 8. Micrographs before and after deposition on top of a three-piece mosaic structure with gaps along the [100] and [110] direction. (a) DICM image of the mosaic after soldering of the substrates on top of a molybdenum plate in which the arrow indicates the [110] direction. (b) DICM image and (c) SEM image (taken at $45^{\circ}$ with the normal at the top face) of the mosaic after flame deposition of one more than $250 \mu \mathrm{m}$ thick single crystal diamond layer on top of it. (d), (e) Continuous macrostep patterns on the deposited diamond layer respectively across the [110] and [j00] joint region; the dark band on the photographs along the direction of the arrows are the out of focus images of the initial gaps below the surface. Images (a), (b) and (c) are on the same scale just like images (d) and (e). 
maximum height of about $50 \mu \mathrm{m}$. However, none of the observed features show any discontinuity across the joint region (see Fig. 7b) nor resembles any correlation with the band of enhanced growth parallel to the initial gap which was obtained by hot filament growth. Either this bunch was etched away and overgrown during flame deposition or completely "pushed" out of the specimens top face. Although sub-surface inspection of the specimen by optical microscopy is hindered by the increased surface roughness, the initial gap between the substrates could be observed at about $265 \mu \mathrm{m}$ below the surface as indicated by the arrow in Fig. 7c. Even the original zigzag crack pattern confined to the limited thickness of the HFCVD film which crosses this gap several times can be identified above the arrow at the top of this figure. The diamond peaks in several Raman spectra obtained from the joint region in average have the same FWHM of $2.8 \mathrm{~cm}^{-1}$ as the peaks in spectra from other parts of the specimen, but show a slight shift in position from 1332.3 to $1332.6 \mathrm{~cm}^{-1}$.

In a next experiment, a three-piece mosaic cut from a (001)-oriented type IIa natural diamond plate with one sloping side and gaps in two different directions, viz. [100] and [110], was overgrown directly by the acetylene-oxygen flame (see Fig. 8). The plate had a misorientation of $3^{\circ}$ towards [100], the same direction as used for the previous described mosaics which were successfully overgrown. The steps at this misoriented surface are fully kinked and expected to propagate faster than stable $\langle 110\rangle$-oriented steps, which might be advantageous in closing the gaps between the different elements of the mosaic. Two small bevels or alignment facets were polished in the corners of the plate to recognize the otherwise identical triangular elements of the mosaic after laser cutting. Following the cutting procedure, the cut side faces were repolished. By this procedure the side faces on both sides of a gap have the same orientation within $\pm 0.2^{\circ}$. Fig. 8a shows the mosaic after soldering of the elements upon a molybdenum plate. No precautions were taken to prevent the pieces floating apart during brazing. Inspection by DICM prior to growth revealed that the width of the gap along [100] from one side to the other roughly varied from 15 to $25 \mu \mathrm{m}$, the gap along [110] varied from 7 to $25 \mu \mathrm{m}$. Due to this change in width of the gaps, the in-plane crystallographic alignment of the seed crystals with respect to each other was within $\pm 0.6^{\circ}$. The alignment was estimated to be within $\pm 0.2^{\circ}$ in both other perpendicular directions, as could be deduced by the small differences in hight $(<1 \mu \mathrm{m})$ between the substrates on both sides of the gaps and uniformity in colour of the substrate top faces as observed by DICM. Because the misalignment of the substrates is within the $0.5^{\circ}$ to $1^{\circ}$ line width of natural type Ila diamond [50], the X-ray diffraction pattern of the mosaic did not reveal any double spots.

The obtained mosaic structure was thoroughly etched for half an hour at $975^{\circ} \mathrm{C}$ and $S_{\text {ac }}=-2 \%$. This procedure was directly followed by $11 \mathrm{~h}$ of growth at $1030^{\circ} \mathrm{C}$ and a gradually increased supersaturation from $4 \%$ to $5 \%$. Figs. $8 \mathrm{~b}-8 \mathrm{e}$ show the result of this experiment. The intensive etch procedure appeared successful because the deposited layer of about $250 \mu \mathrm{m}$ in thickness exhibits no hillocks originating from foreign particles or cusps. The [100] misorientation of the substrates has dictated the direction of the macrosteps on the largest part of the top face. A small part of the top face along the sloping side of the mosaic has become exactly oriented during growth and is dominated by energetically favourable steps along $\langle 110\rangle$. As was also observed for the former described mosaic, the exact and misoriented parts of the top face are separated by a pronounced step bunch, which in this case has a maximum height of about $23 \mu \mathrm{m}$ (see Fig. 8c). Preliminary analyses seem to indicate that the development of these relatively large step bunches is related with the misorientation of the substrates in a $\langle 100\rangle$ direction. The surface reveals no bands of extended growth originating from the gaps between the substrates of which the positions could be found by focusing into the deposited layer with an optical microscope. Figs. 8d and 8 e respectively show the surface morphology of the diamond layer above the [110]- and [100]-oriented gaps in the areas as indicated in Fig. 8b. The dark shadows running from top to bottom of Figs. 8d and 8e indicated by the arrows, are the out-focus images of the original gaps located about $250 \mu \mathrm{m}$ below the surface. From the fact that the complex macrostep patterns of the top layer cross the joint region unhindered, a feature which was observed along the full length of both joint regions, 
it is clear that the three pieces of the original mosaic have become one single crystal. In an early stage of the growth experiment several dark lines, indicative for crack formation, were observed at the left-hand piece of the mosaic. These lines faded more and more after prolonged deposition. By post-growth sub-surface inspection of the mosaic with an optical microscope, indeed two large parallel cracks along \{111\} cracking planes were found. These cracks, which end at the original [100] gap, do not proceed up to the surface of the grown layer, which above the cracks reveals the same continuous step patterns as above the initial gaps between the substrates.

As expected from the original misorientation of the substrates, CL topography in the largest part of the specimen mainly reveals orange-red emission of the $575 \mathrm{~nm}$ system. Although far less in intensity, this emission still dominates in the more exactly oriented part of the surface for which the macrosteps are more or less oriented along the perpendicular $\langle 110\rangle$ directions. Surprisingly the surface reveals bands of blue $\mathrm{CL}$ aligned along [110] above the cracks and the initial [110] gap of the mosaic. A similar feature observed in small $\{001\}$-oriented crystallites was reported in the literature [51]. The occurrence of this emission can be explained by the presence of dislocation lines which, in case of relatively low nitrogen concentrations, show violet-blue CL polarized with the $E$-vector parallel to the dislocation line $[39,40]$. High magnification observation of pronounced line-shaped blue emission centra through a linear polarizing filter indeed indicates a polarization of the luminescence. This is strong evidence for the statement that band A luminescence in flame-grown diamond is dislocation related (see Section 2). Raman spectra taken from regions of the top face away from the joint have a diamond peak at $1332.0 \mathrm{~cm}^{-1}$ with a FWHM of $3.0 \mathrm{~cm}^{-1}$. The diamond Raman peak in the spectrum obtained from the $[110]$ joint region is located at the same position but has a FWHM of $3.3 \mathrm{~cm}^{-1}$, while that from the [100] joint has a FWHM of $3.0 \mathrm{~cm}^{-1}$ but is shifted to $1332.3 \mathrm{~cm}^{-1}$. One might argue that the increased dislocation density in the [110] joint region as indicated by CL topography increases the FWHM of the diamond Raman peak somewhat but also allows for the release of stress induced by the joining of two not perfectly aligned substrates, which at the [100] joint causes a shift in the position of the peak. The higher density of dislocations in the [110] joint can be explained by the fact that closure of a $\langle 110\rangle$-oriented gap, at least partly occurs by growth via the \{111\} sectors which are more defective than others.

\section{Discussion and conclusions}

The morphology of cubic diamond faces obtained by homoepitaxial acetylene-oxygen flame deposition, is determined by macrosteps or bunch formations caused by step pinning and piling-up of lower steps. It was argued that the presence of nitrogencontaining species in the gas phase has a significant influence on the occurrence of these phenomena. Although based on previous Raman and IR analyses, diamond volumes grown via the $\{001\}$ faces using the flame technique must be assigned as type IIa, CL spectroscopy usually reveals blue band $\mathrm{A}$ as well as the orange-red $575 \mathrm{~nm}$ emission indicating a small amount of incorporated nitrogen [2]. CL topography shows that this as $\mathrm{N}-\mathrm{V}$ incorporated nitrogen is not uniformly distributed over the $\{001\}$ faces, which could be explained by a difference in step geometry. Macrosteps along the perpendicular $\langle 110\rangle$ directions are smooth and stable so the uptake of nitrogen impurities in these steps is less than for macrosteps along other directions which are rough and on an atomic scale have a higher density of kink sites. The observation of linear-polarized blue CL strongly indicates that band $\mathrm{A}$ emission in flame-grown diamond is dislocation related.

Bundles of dislocations, foreign (diamond) particles and imperfections at the substrate edges, in particular cusps, were found to be highly active sources of steps on the $\{001\}$ faces of the flame-grown diamond single crystals. These growth centres cause elevations and give rise to relatively large crystallographic imperfections in the diamond layer. Owing to the non-uniform distribution of the growth centres and the specific geometry of the combustion flame gas phase, the cubic top faces of the obtained single crystals tend to have a concave shape. Pre-growth etching of the natural diamond seed crystals in the oxidizing outer zone of the acetylene-oxygen flame resulted in homoepitaxial diamond layers with an improved crystallographic quality. This is because 
preferred etching of the highly active growth centres enables a more uniformly distributed step generation by the large number of individual dislocations present in natural type IIa diamond seed crystals. It was demonstrated that even cracks and hillocks on HFCVD-grown layers can be overgrown by a homogeneous flame-grown layer after an initial etch procedure.

The most direct way to enlarge the dimensions of single crystal diamond substrates needed for future optoelectronic devices, is to overgrow a mosaic structure of several $\{001\}$-oriented diamond substrates by the deposition of one single crystal diamond layer. Due to the decrease of crystallographic imperfections especially at the edges of the flamegrown cubic faces by pre-growth etching, it became possible to join such mosaic structures by the flame technique. The present work is the first report in which a mosaic structure with gaps along $\langle 100\rangle$ as well as along $\langle 110\rangle$ was overgrown. The single crystalline nature of the deposited layer could be confirmed by the observation of continuous growth step patterns on the diamond layer across the joint region. The widths of the gaps were between 7 and $25 \mu \mathrm{m}$ which is more than an order of magnitude larger than those along $\langle 100\rangle$, of which the single crystal overgrowth by HFCVD evidenced by crack patterns crossing the joint region was previously reported [11]. Furthermore the present study demonstrated that the side faces at both sides of the gap do not necessarily need to be parallel. This and the increase in gap width that could be bridged is of great importance because it allows small in-plane rotations of the individual mosaic substrates of which the side faces never have exactly the same crystallographic orientation. The fact that both the gaps along $\langle 100\rangle$ and along $\langle 110\rangle$ can be overgrown indicate that most probably gaps along any direction can be overgrown. So a large variety of substrate shapes might be used to be arranged in a mosaic structure. Micro-Raman analyses of the overgrown mosaics indicate that the increased dislocation density of $\langle 110\rangle$ joint regions might allow relaxation of strain at the joint. Nevertheless, a joint along $\langle 110\rangle$ is less favourable because this direction is parallel to the $\{111\}$ cracking plane of diamond and involves the risk of fracturing the overgrown layer along the joint. Furthermore, the development of defective $\{111\}$ facets in these $\langle 110\rangle$ gaps can obstruct the formation of one single crystalline diamond layer on top of the mosaic structure. The incorporation of small amounts of nitrogen in flame-grown layers does not form a problem to apply this technique for the growth of large single crystal substrates for diamond-based devices. This is because the deposition of such structures, which must be done by more gentle techniques like HFCVD or MWCVD, can be preceded by growth of a nitrogen-free buffer layer. In fact, preliminary results of our present investigations indicate that the addition of a small amount of $\mathrm{NH}_{3}$ during homoepitaxial diamond growth by HFCVD reduces the formation of cracks in the deposited layers [55].

\section{Acknowledgements}

The authors wish to thank Dr. G. Janssen for providing the HFCVD pre-grown specimen and helpful discussions. The photo-service department is acknowledged for the careful processing of the photographs. This work was financially supported by the European Community Brite Euram project BE 5099 . 92 under contract BRE-2-0147.

\section{References}

[1] L.F. Sutcu, C.J. Chu, M.S. Thompson, R.H. Hauge, J.L. Margrave and M.P. D’Evelyn, J. Appl. Phys. 71 (1992) 5930.

[2] J.J. Schermer, W.J.P. van Enckevort and L.J. Giling, Diamond Related Mater. 3 (1994) 408.

[3] G. Janssen, W.J.P. van Enckevort, W. Vollenberg and L.J. Giling, J. Crystal Growth 148 (1995) 355.

[4] W.J.P. van Enckevort, G. Janssen, W. Vollenberg and L.J. Giling, J. Crystal Growth 148 (1995) 365.

[5] X. Jiang, C.-P. Klages, R. Zachai, M. Hartweg and H.-J. Füsser, Appl. Phys, Lett. 62 (1993) 3438.

[6] C. Wild, R. Kohl, N. Herres, W. Müller-Sebert and P. Koidl, Diamond Related Mater. 3 (1994) 373.

[7] G.Z. Cao, J.J. Schermer, W.J.P. van Enckevort, W.A.L.M. Elst and L.J. Giling, J. Appl. Phys. 79 (1996) 1357.

[8] B.R. Stoner and J.T. Glass, Appl. Phys. Lett. 60 (1992) 698.

[9] M.W. Geis, H.I. Smith, A. Argoitia, J. Angus, G.-H.M. Ma, J.T. Glass, J. Butler, C.J. Robinson and R. Pryor, Appl. Phys. Lett. 58 (1991) 2485.

[10] M.W. Geis, N.N. Efremow, R. Susalka, J.C. Twichell, K.A. Snail, C. Spiro, B. Sweeting and S. Holly, Diamond Related Mater. 4 (1994) 76. 
[11] G. Janssen and L.J. Giling, Diamond Related Mater. 4 (1995) 1025.

[12] D.P. Malta, J.B. Posthill, G.C. Hudson, R.E. Thomas, T.P. Humphreys, R.A. Rudder and R.J. Markunas, in: Proc. 4th Int. Symp. on Diamond and Related Mater., Eds. K.V. Ravi and J.P. Dismukes (Electrochemical Society, Pennington, NJ, 1995) p. 509.

[13] N.R. Parikh. J.D. Hunn, E. McGucken, M.L. Swanson, C.W White, R.A. Rudder, D.P. Malta, J.B. Posthill and R.J Markunas, Appl. Phys. Lett. 61 (1992) 3124

[14] Y. Tzeng, J. Wei, J.T. Woo and W. Lanford, Appl. Phys. Lett. 63 (1993) 2216.

[15] M. Marchywka, P.E. Phersson, D.J. Vestyck, Jr. and D. Moses, Appl. Phys. Lett. 63 (1993) 3521.

[16] J.J. Schermer, J.E.M. Hogenkamp, G.C.J. Otter, G. Janssen, W.J.P. van Enckevort and L.J. Giling, Diamond Related Mater. 2 (1993) 1149.

[17] J.J. Schermer and L.J. Giling, in: Proc. 4th Int. Symp. on Diamond and Related Mater., Eds. K.V. Ravi and J.P. Dismukes (Electrochemical Society, Pennington, NI, 1995) $\mathrm{p}$ 212.

[18] K. Okada. S. Komatsu, S. Matsumoto and Y. Moriyoshi, J Crystal Growth 108 (1991) 416.

[19] D.P. Malta, J.B. Posthill, R.A. Rudder, G.C. Hudson and R.J Markunas, J. Mater. Res. 8 (1993) 1217.

[20] K.A. Snail, C.L. Vold, C.M. Marks and J.A. Freitas, Jr., Diamond Related Mater. 1 (1992) 180

[21] W.J.P. van Enckevort, G. Janssen, W. Vollenberg, J.J. Schermer and L.J. Giling, Diamond Related Mater. 2 (1993) 997.

[22] J.J. Schermer, P. Alers and L.J. Giling, J. Appl. Phys. 78 (1995) 2376.

[23] W.J.P. van Enckevort, G. Janssen, J.J. Schermer and L.J. Giling, Diamond Related Mater. 4 (1995) 250.

[24] J.P. van der Eerden and H. Müller-Krumbhaar, Electrochim. Acta 31 (1986) 1007.

[25] F.P. Bundy. H.M. Strong and R.H. Wentorf, in: Chemistry and Physics of Carbon, Vol. 10, Eds. P.L. Walker and P.A Thrower (Dekker, New York, 1973) p. 213.

[26] J.J. Schermer, W.A.L.M. Elst and L.J. Giling, Diamond Related Mater. 4 (1995) 1113.

[27] R. Locher, C. Wild, N. Herres, D. Behr and P. Koidl, Appl Phys. Lett. 65 (1994) 34.

[28] P. Hartman and W.G. Perdok, Acta Cryst. 8 (1955) 49, 521 525.

[29] L.J. Giling and W.J.P. van Enckevort, Surf. Sci. 161 (1985) 567.

[30] J.J. Schermer, W.J.P. van Enckevort and L.J. Giling, J Crystal Growth 148 (1995) 248.
[31] P.J. Dean, Phys. Rev. 139 (1965) A588

[32] L.H. Robins, L.P. Cook, E.N. Farabaugh and A. Feldman, Phys. Rev. B 39 (1989) 13367.

[33] A.T. Collins, M. Kamo and Y. Sato, J. Phys. D 22 (1989) 1402.

[34] G. Davies, Chem. Phys. Carbon 13 (1977) 1.

[35] R.M. Chrenko, Phys. Rev. B 7 (1973) 4560.

[36] E.C. Lightowlers and A.T. Collins, J. Phys. D 9 (1976) 951

[37] V.S. Vavilov, A.A. Gippius, A.M. Zaitsev, B.V. Deryagin B.V. Spitsyn and A.E. Aleksenko, Sov. Phys. Semicond. 14 (1980) 1078

[38] R.J. Graham, T.D. Moustakas and M.M. Disko, J. Appl. Phys. 69 (1991) 3212.

[39] I. Kiflawi and A.R. Lang, Philos. Mag. 33 (1976) 697

[40] P.L. Hanley, I. Kiflawi and A.R. Lang, Philos. Trans. R. Soc. London A 284 (1977) 329.

[41] A.G. Gaydon and H.G. Wolfhard, Flames: Their Structure, Radiation and Temperature (Chapman \& Hall, London, 1979).

[42] K.A. Snail and C.J. Craigie, Appl. Phys. Lett. 58 (1991) 1875

[43] J.E. Butler and R.L. Woodwin, Philos. Trans. R. Soc. London A 342 (1993) 209.

[44] R.J.H. Klein-Douwel, J.J.L. Spaanjaars and J.J. ter Meulen, J. Appl. Phys. 78 (1995) 2086.

[45] R.E. Clausing, L. Heatherly, L.L. Horton, E.D. Specht, G.M. Begun and Z.L. Wang, Diamond Related Mater. 1 (1992) 411

[46] M. Kamo, H. Yurimoto and Y. Sato. Appl. Surf. Sci. 33/34 (1988) 553

[47] J.P. Vitton, J.J. Garenne and S. Truchet, Diamond Related Mater. 2 (1993) 713.

[48] H. Klapper, in: Characterisation of Crystal Growth Defects by X-Ray Methods, Eds. B.K. Tanner and D.K. Bowden (Plenum, New York, 1980) p. 133.

[49] N. Lee and A. Badzian, Appl. Phys. Lett. 66 (1995) 2203.

[50] J.E. Field. Ed., The Properties of Natural and Synthetic Diamond (Academic Press, London, 1992).

[51] N.C. Burton, J.E. Butler, A.R. Lang and J.W. Steeds, Proc. R. Soc. London A 449 (1995) 555.

[52] C. Wild. P. Koidl, W. Müller-Sebert, H. Walcher, R. Kohl, N. Herres, R. Locher, R. Samlenski and R. Brenn. Diamond Related Mater. 2 (1993) 158.

[53] B.A. Fox, B.R. Stoner, D.M. Malta, P.J. Ellis, R.C. Glass and F.R. Sivazlian, Diamond Related Mater. 3 (1994) 382.

[54] Drukker Int. B.V., Cuijk, The Netherlands.

[55] J.J. Schermer and G.Z. Cao, work in progress. 